\title{
You Assume, We Guarantee: Methodology and Case Studies*
}

\author{
Thomas A. Henzinger Shaz Qadeer Sriram K. Rajamani \\ EECS Department, University of California at Berkeley, CA 94720-1770, USA \\ Email:\{tah, shaz,sriramr\}@eecs . berkeley.edu
}

\begin{abstract}
Assume-guarantee reasoning has long been advertised as an important method for decomposing proof obligations in system verification. Refinement mappings (homomorphisms) have long been advertised as an important method for solving the languageinclusion problem in practice. When confronted with large verification problems, we therefore attempted to make use of both techniques. We soon found that rather than offering instant solutions, the success of assume-guarantce reasoning depends critically on the construction of suitable abstraction modules, and the success of refinement checking depends critically on the construction of suitable witness modules. Morcover, as abstractions need to be witnessed, and witnesses abstracted, the process must be iterated. We present here the inain lessons we learned from our experiments, in form of a systematic and structured discipline for the compositional verification of reactive modules. An infrastructure to support this discipline, and automate parts of the verification, has been implemented in the tool MOCHA.
\end{abstract}

\section{Introduction}

Formal verification is a systematic approach for detecting logical errors in designs. The designer uses a language with mathematical semantics to describe the design, which is then analyzed for correctness with respect to a specification. We refer to the design being analyzed as the implementation. The verification problem is called refinement checking when the specification is a more abstract design. For a trace semantics, the refinement-checking problem is PSPACEhard in the size of the implementation description and in the state space of the specification. Not surprisingly, algorithms for refinement checking are exponential in the size of the implementation description and doubly exponential in the size of the specification description.

There are two general classes of techniques for combating this state-explosion problem. Type- 1 techniques focus on improving algorithms, often developing heuristics that target specific application domains, such as symbolic methods for synchronous hardware designs, and partialorder methods for asynchronous communication protocols. Type- 2 techniques focus on dividing the verification task at hand into simpler tasks, often making use of the compositional structure of both implementation and specification, such as assume-guarantee methods for proof decomposition. While type- I techniques can be applied fully automatically and improve the efficiency of formal verification, they need to be complemented by type-2 techniques in order to make the approach fully scalable. Type- 2 techniques, however, require substantial assistance from human verification experts, and their systematic application in nontrivial situations remains somewhat of a black art.

We are developing a formal-verification tool, called МоснA [AHM ${ }^{+98}$ ], which is based on the system description language of reactive modules [AH96]. Rcactive modules permit the modular and hicrarchical description of heterogencous systems, and have been designed explicilly to support type-2 techniques such as assume-guarantee reasoning. In this paper, we present the

\footnotetext{
" This work is supported in part by ONR YIP award N00014-95-1-0520, by NSF CAREER award CCR9501708, by NSF grant CCR-9504469, by ARO MURI grant DAAH-04-96-1-0341, and by the SRC contract 97-DC-324.041.
} 
experiences and results of our attempts to make use of type- 2 techniques within MocHA in a disciplined and systematic way. We report on a methodology that has led us to success in verifying a hardware circuit that implements Tomasulo's algorithm, and a sliding-window communication protocol. Since the description of these examples would require more space than is available in these procecdings, we illustrate our methodology, instead, on a circuit that implements a simple threc-stage pipeline.

We now briefly outline our methodology, which approaches a refinement-checking problem of the form $P_{1} \| P_{2} \preceq Q$ (where $\preceq$ is the trace-containment relation) by introducing abstraction and witness modules. Suppose that the state space of the implementation $P_{1} \| P_{2}$ is too large to be handled by exhaustive search algorithms. A naive compositional approach would attempt to prove both $P_{1} \preceq Q$ and $P_{2} \preceq Q$, and then conclude $P_{1} \| P_{2} \preceq Q$. Though sound, the naive approach often fails in practice, because $P_{1}$ usually refincs $Q$ only in a suitable constraining environment, and so does $P_{2}$. Hence we construct a suitable constraining environment $A_{2}$ for $P_{1}$, and similarly $A_{1}$ for $P_{2}$. Since $A_{1}$ describes the aspects of $P_{1}$ that are relevant to constraining $P_{2}$, and similarly $A_{2}$ is an abstract description of $P_{2}$, the two new modules $A_{1}$ and $A_{2}$ are called abstraction modules. By assume-guarante reasoning, we conclude $P_{1} \| P_{2} \preceq Q$ from the two proof obligations $P_{1}\left\|A_{2} \preceq A_{1}\right\| Q$ and $A_{1}\left\|P_{2} \preceq Q\right\| A_{2}$.

Traditionally, the size of the implementation has been viewed as the main source of complexity for the refinement-checking problem. In our approach, we shift the focus to the size of the gap between the implementation and the specification. As an extreme case, if we are given two identical copies of a design, we ought to be able to verify that one is a valid refinement of the other, no matter how large the designs. We want the success rate of our methodology to increase if the designer invests effort in structuring the implementation and specification so as to expose more commonality between them. Abstraction modules form an intermediate layer between the implementation and the specification, and thus provide a systematic way of reducing the gap. In our case studies, we found that abstraction modules generally take the form of abstract definitions for hidden implementation variables. When composed with the original specification, which often specifies only relationships between primary inputs and outputs, the abstraction modules yield a richer specification that is closer to the implementation. Constructing good abstraction modules requires manual effort. Once constructed, our methodology automatically makes effective use of the abstraction modules to decompose the refinement check.

Even if the state space of the implementation becomes manageable as a result of proof decomposition, each remaining refinement check, say $P^{\prime}=P_{1}\left\|A_{2} \preceq A_{1}\right\| Q=Q^{\prime}$, is still PSPACEhard in the size of the specification state space. However, for the special ease that all variables of $Q^{\prime}$ are also present in $P^{\prime}$ (in this case, we say that $Q^{\prime}$ is projection refinable by $P^{\prime}$ ), the re. finement check reduces to a transition-invariant check, which verifies that every move of $P^{\prime}$ can be mimicked by $Q^{\prime}$. The complexity of this procedure is linear on the state spaces of both $P^{\prime}$ and $Q^{\prime}$. If $Q^{\prime}$ is not projection refinable by $P^{\prime}$, our methodology advocates the introduction of a witmess module $W$, which makes explicit how the hidden variables of the specification $Q^{\prime}$ depend on the state of the implementation $P^{\prime}$. Then $Q^{\prime}$ is projection refinable by $P^{\prime} \| W$, and it suffices to prove $P^{\prime} \| W \preceq Q^{\prime}$ in order to conclude $P^{\prime} \preceq Q^{\prime}$. The construction of witness modules also requires manual effort, but whenever the specification $Q^{\prime}$ simulates the implementation $P^{\prime}$, a suitable witness can be found.

Related work. The individual pieces of our methodology are not new; we simply advocate their disciplined use within the framework of reactive modules. In particular, assume-guarantee rules for various formalisins can be found in [Sta85,CLM89,GL94,AL95,McM97]; the rule used in this paper has been taken from [AH96]. Witnesses have appeared in various guises and forms (homomorphisms, refinement mappings, simulation relations, etc.) in different works [Lam83,LT87,AL91,BBLS92,CGL92,Kur94,LV95,McM97]. Also our choice of case studies is 
not new. Other correctness proofs for Tomasulo's algorithm can be found in [DP97,McM98]; the sliding-window protocol is taken from [Tan92].

\section{A Verification Problem}

Reactive modules. Reactive modules is a formalism for the modular description of systems with heterogeneous components. The definition of reactive modules can be found in [AH96]; here we give only a brief introduction. The state of a reactive module is determined by the values of threc kinds of variables: the external variables are updated by the environment and can be read by the module; the interface variables are updated by the module and can be read by the environment; the private variables are updated by the module and cannot be read by the environment. The external and interface variables are called observable; the interface and private variables, controlled.

The state of a reactive module changes in a scquence of rounds. The first round is called the initial mund, and determines initial values for all variables. Each subsequent round is called an update round, and determines new values for all variables. For external variables, the values in the initial and update rounds are left unspecified (i.e., chosen nondeterministically). For controlled variables, the values in the initial and update rounds are specified by (possibly nondeterministic) guarded commands. In each update round, the new value of a controlled variable may depend on the (latched) values of some variables from the previous round. In addition, in each round, the initial (or new) value of a controlled variable may depend on the initial (or new) values of some other variables from the same round; such a dependency between the values of variables within a single round is called an await dependency. In order to avoid inconsistent specifications, the await dependencies must be acyclic. In reactive modules, the acyclicity restriction is enforced statically, by partitioning the controlled variables into atoms that can be ordered such that in cach round, the initial (or new) values for all variables of an atom can be determined simultancously from the initial (or new) values of the external variables and the variables of earlier atoms.

Each round, therefore, consists of several subrounds-one for the external variables, and one per atom. Each atom has an initial commend, which specifies the possible initial values for the variables of the atom, and an update command, which specifies the possible new values for the variables of the atom within each update round. In the update command, unprimed occurrenees of variables refer to the latched values from the previous round; in both the initial and update commands, primed occurrences of variables refer to the initial (or new) values from the same round.

Example 1. Consider the simple instruction set architecture defined by the reactive module ISA of Figure 1. The module ISA has five external variables (inputs) - the operation op, the immediate operand inp, the source registers srcl and src2, and the destination register dest. There are two interface variables (oulputs) - the value out of a STORE instruction, and a boolean variable stall, which indicates if the current inputs have been accepted. If the value of stall is true in a round, then no instruction is processed in that round, and the environment is supposed to produce the same instruction again in the next round. Finally, there is one private variable-the register file isaRegFile.

A round of the module ISA consists of four subrounds. In the first subround of each update round, the environmenl chooses an operation, operands, and a destination, by assigning values to the external variables. In the second subround, the atom ISAStall decides nondeterministically if the current inputs are processed, by setting stall to true or false. The third subround belongs to the atom ISARegFile. If the updated value of stall is false, then the current instruction is processed appropriately. If the operation is $A N D$ or $O R$, it is performed on the source registers and the result is placed into the destination register. If the operation is $L O A D$, the immediate operand is assigned to the destination register. The fourth subround belongs to the atom ISAOut. If the 


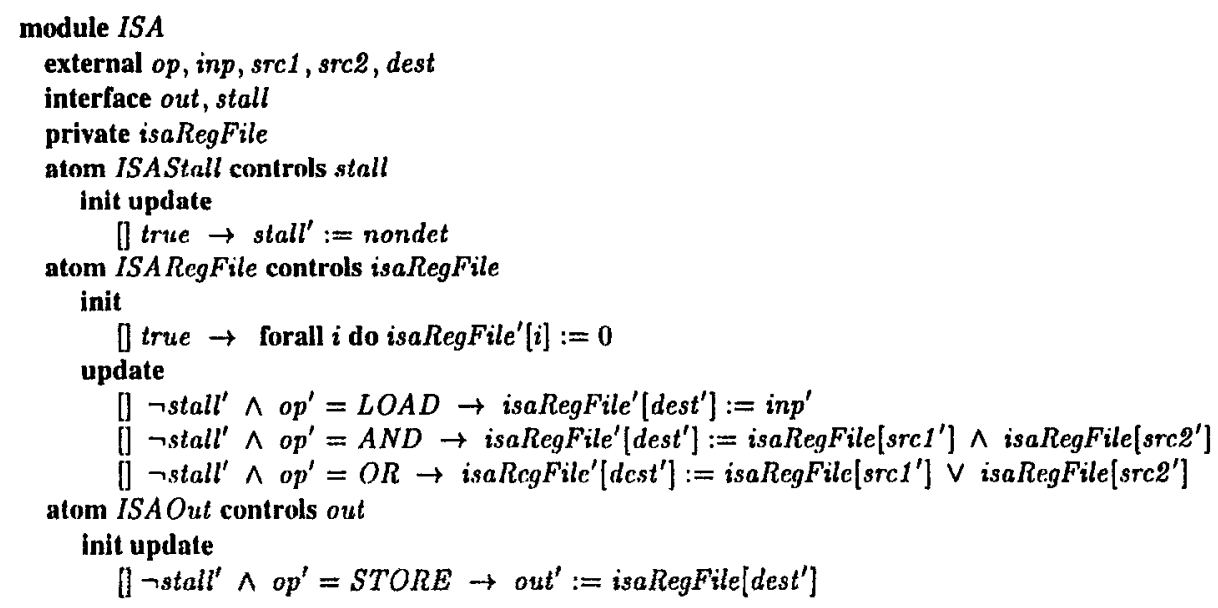

Fig. 1. Instruction set architecture

updated value of stall is false and the current operation is STORE, then out is updated to the contents of the destination register from the previous round. Since both atoms ISARegFile and ISAOut wait, in each update round, for the new value of stall, they must be executed after the atom ISAStall, which produces the new value of stall. However, there are no await dependencies between the atoms ISARegFile and ISAOnt, and therefore the third and fourth subrounds of each update round can be interchanged.

Parallel composition. The composition operation combines two reactive modules into a single module whose behavior captures the interaction between the two component modules. Two modules $P$ and $Q$ are compatible if (1) the controlled variables of $P$ and $Q$ are disjoint, and (2) the await dependencies between the variables of $P$ and $Q$ are acyclic. If $P$ and $Q$ are two compatible modules, then the composition $P \| Q$ is the module whose atoms are the (disjoint) union of the atoms from $P$ and $Q$. The interface variables of $P \mid i Q$ are the (disjoint) union of the interface variables of $P$ and $Q$, and the private variables of $P \| Q$ are the (disjoint) union of the private variables of $P$ and $Q$. The external variables of $P \| Q$ consist of the external variables of $P$ that are not interface variables of $Q$, and the external variables of $Q$ that are not interface variables of $P$.

Example 2. The module $I S A$ from Figure 1 can be seen as the parallel composition of three modules. The module ISAStall has the interface variable stall; the module ISARegFile has the external variables op, inp, src1, src2, dest, and stull, and the interface variable isaRegFile; the module ISAOut has the external variables op, dest, stall, and isaRegFile, and the interlace variable out. The opcration hide makes the interface variable isaRegFile private:

$$
I S A=\text { hide isaRegFile in ISAStall||ISARegFile } \mid I S A O u t
$$

Refinement. The notion that two reactive modules describe the same system at different levels of detail is captured by the refinement relation between modules. We define refinement as trace containment. A state of a module $P^{\prime}$ is a valuation for the variables (external, interface, and private) of $P$. A state is initial if it can be obtained at the end of the initial round. Given two states $s$ and $t$, we write $s \rightarrow_{P} t$ if when the state at the beginning of an update round is $s$, then the state at the end of the update round may be $t$. A trajectory of $P$ is a finite sequence $s_{0}, \ldots, s_{n}$ of states such that (1) $s_{0}$ is an initial state of $P$, and (2) for $i \in\{0,1, \ldots, n-1\}$, 


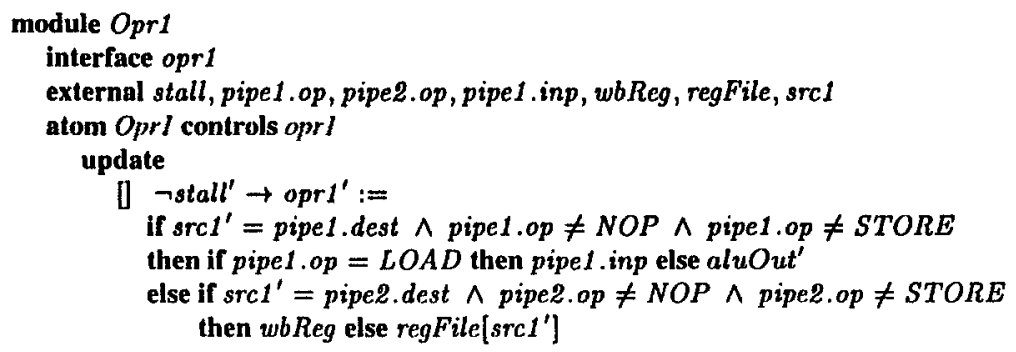

module Opr2

interface opr2

external stall, pipe1 .op, pipe2 .op, pipe1 inp, wbReg, regFile, src2

"Same as $O p r 1$ with $\operatorname{src} 1$ replaced by $s r c 2$ "

module Pipe1

Interface pipe1.op, pipe1.inp, pipe1.dest

external stall, inp, op, dest

atom Pipe1 controls pipe1.op, pipe1.dest, pipe1.inp init

[ true $\rightarrow$ pipe1.op' $:=$ NOP

update

[] true $\rightarrow$ pipel.op' $:=$ if stall' then NOP else op'; pipe1.dest' $:=$ dest $^{\prime} ;$ pipe1.inp' $:=$ inp' $^{\prime}$

Fig. 2. Pipeline stage 1

we have $s_{i} \rightarrow P s_{i+1}$. The states that lie on trajectories are called reachable. An observation of $P$ is a valuation for the observable variables (external and interface) of $P$. If $s$ is a valuation to a set of variables, we use $[s]_{P}$ to denote the set of valuations from $s$ restricted to the observable variables of $P$. For a state sequence $\bar{s}=s_{0}, \ldots, s_{n}$, we write $[\bar{s}]_{P}=\left[s_{0}\right]_{P}, \ldots,\left[s_{n}\right]_{P}$ for the corresponding observation sequence. If $\bar{s}$ is a trajectory of $P$, then the projection $[\bar{s}]_{P}$ is called a trace of $P$. The module $Q$ is refinable by module $P$ if (1) every interface variable of $Q$ is an interface variable of $P$, and (2) every external variable of $Q$ is an observable variable of $P$. The modulc $P$ refines the module $Q$, written $P \preceq Q$, if (1) $Q$ is refinable by $P$, and (2) for cvery trajectory $\bar{s}$ of $P$, the projection $[\bar{s}]_{Q}$ is a trace of $Q$.

Example 3. Consider the three-stage pipeline defined by the reactive module PIPELINE shown in Figures 2 and 3 . In the first stage of the pipeline, the operands are fetched; in the second stage, the operations are performed; in the third stage, the result is written into the register file. The PIPELINE module is the parallel composition of seven modules. The first stage consists of the modules Pipe1, Opr1, and Opr2. Forwarding logic in Opr1 and Opr2 ensures that correct values are given to the second stage, even if the value in question has not yet been written into the register file. The second stage consists of the module Pipe2, which has an $A L U$ atom that processes arithmetic operations using the operands from the first stage and writes the results into a write-back register called wbReg. The third stage is consists of the module RegFile, which copies wbReg into the appropriate register. The PipeOut module outputs a register value in response to a STORE instruction. The Stall module controls the stall signal, which is set to true whenever a STORE instruction cannot be accepted due to data dependencies. 
Our goal is to show that PIPELINE is a correct implementation of the instruction set architecture ISA. This is the case if every sequence of instructions given to PIPELINE produces a sequence of outputs (and stalls) that is permitted by $I S A$. The module ISA is refinable by PIPELINE, so it remains to be shown that cvery trace of PIPELINE is a trace of ISA.

\section{Our Methodology}

Witness modules. The problem of checking if $P \preceq Q$ is PSPACE-hard in the state space of $Q$. However, the refinement check is simpler in the special case in which all variables of $Q$ are observable. The module $Q$ is projection refinable by the module $P$ if (1) $Q$ is refinable by $P$, and (2) $Q$ has no private variables. If $Q$ is projection refinable by $P$, then every variable of $Q$ is observable in both $P$ and $Q$. Therefore, checking if $P \preceq Q$ reduces to checking if for every trajectory $\bar{s}$ of $P$, the projection $[\bar{s}]_{Q}$ is a trajectory of $Q$. According to the following proposition, this can be donc by a transition-invariant check, whose complexity is lincar in the state spaces of both $P$ and $Q$.

Proposition 1. [Projection refinement] Consider two modules $P$ and $Q$, where $Q$ is projection refinable by $P$. Then $P \preceq Q$ iff $(1)$ if $s$ is an initial state of $P$, then $[s]_{Q}$ is an initial state of $Q$, and (2) if $s$ is a reachable state of $P$ and $s \rightarrow_{P} t$, then $[s]_{Q} \rightarrow_{Q}[t]_{Q}$.

We make use of this proposition as follows. Suppose that $Q$ is refinable by $P$, but not projection refinable. This means that there are some private variables in $Q$. Define $Q^{u}$ to be the module obtained by making every private variable of $Q$ an interface variable. If we compose $P$ with a module $W$ whose interface variables include the private variables of $Q$, then $Q^{u}$ is projection refinable by the composition $P \| W$. Morcover, if $W$ docs not constrain any external variables of $P$, then $P \| W \preceq Q^{u}$ implies $P \preceq Q$ (in fact, $P$ is simulated by $Q$ ). Such a module $W$ is called a witness to the refinement $P \preceq Q$. The following proposition states that in order to check refinement, it is sufficient to first find a witness module and then check projection refinement.

Proposition 2. (Witness modules/ Consider two modules $P$ and $Q$ such that $Q$ is refinable by $P$. Let $W$ be a module such that (I) $W$ is compatible with $P$, and (2) the interface variables of $W$ include the private variables of $Q$, and are disjoint from the external variables of $P$. Then (I) $Q^{u}$ is projection refinable by $P \| W$, and (2) $P \| W \preceq Q^{u}$ implies $P \preceq Q$.

Furthermore, it can be shown that if $P$ does not have any private variables, and $P$ is simulated by $Q$, then a witness to the refinement $P \preceq Q$ does exist. In summary, the creativity required from the human verification expert is the construction of a suitable witness module, which makes explicit how the private state of the specification $Q$ depends on the state of the implementation $P$.

Assume-guarantee reasoning. The state space of a module may be exponential in the size of the module description. Consequently, even checking projection refinement may not be feasible. However, typically both the implementation $P$ and the specification $Q$ consist of the parallel composition of several modules, in which case it may be possible to reduce the problem of checking if $P \preceq Q$ to several subproblems that involve smaller state spaces. The assume-guarantee rule for reactive modules [AH96] allows us to conclude $P \preceq Q$ as long as each component of the specification $Q$ is refined by the corresponding components of the implementation $P$ within a suitable environment. The following proposition gives a slightly generalized account of the assume-guarantec rule.

Proposition 3. [Assume-guarantee rule] Consider two composite modules $P=P_{1}\|\cdots\| P_{m}$ and $Q=Q_{1}\|\cdots\| Q_{n}$, where $Q$ is refinable by $P$. For $i \in\{1, \ldots, n\}$, let $\Gamma_{i}$ be the composition of arbitrary compatible components from $P$ and $Q$ with the exception of $Q_{i}$. If $\Gamma_{i} \preceq Q_{i}$ for every $i \in\{1, \ldots, n\}$, then $P \preceq Q$. 
module Pipe2

interface pipe2.op, pipe2.dest, wbReg, aluOut

external pipe1.op, pipe1 inp, pipe1.dest, opr1, opr2

atom $A L U$ controls aluOut

update

[] pipe1.op $=A N D \rightarrow$ aluOut $:=$ opr1 $\wedge$ opr2

[] pipe1.op $=$ OR $\rightarrow$ aluOut' $:=$ opr1 1 opr2

atom Pipe2 controls pipe2.op, pipe2.dest

init

[] true $\rightarrow$ pipe2.op $:=$ NOP

update

[] true $\rightarrow$ pipe2.op $:=$ pipe1.op; pipe2.dest $t^{\prime}:=$ pipe1.dest

atom $W b R e g$ controls wbReg

update

[] pipe1.op $=A N D \vee$ pipe1.op $=O R \rightarrow w b \operatorname{Reg}^{\prime}:=a l u O u t^{\prime}$

[] pipe1.op $=L O A D \rightarrow$ wbReg' $:=$ pipe1.inp

module RegFile

interface regFile

external pipe2.op, pipe2.dest, wbReg, aluOut

atom RegFile controls regFile

init

[] true $\rightarrow$ forall $i$ do regFile $[i]:=0$

update

[ pipc2.op $=\Lambda N D \vee$ pipe2. $.0 p=O R \vee$ pipe2.op $=L O A D \rightarrow$

forall $i$ do regFile $[i]:=$ if pipe2.dest $=i$ then $w b$ Reg else regFile $[i]$

module PipeOut

interface out

external op, regFile, dest

atom $O u t$ controls out

update

[] $\neg$ stall' $\wedge o p^{\prime}=S T O R E \rightarrow o u t^{\prime}:=$ regFile[dest']

module Stall

interface stall

external op, dest, pipe1.op, pipe1.dest, pipe2.op, pipe2.dest

atom Stall controls stall

update

$\|$ op ${ }^{\prime}=$ STORE $\wedge$ pipel.op $\neq$ NOP $\wedge$ pipe1.op $\neq$ STORE $\wedge$ dest $=$ pipe1.dest $\rightarrow$ stall' $:=$ true

0 $o p^{\prime}=$ STORE $\wedge$ pipe2.op $\neq$ NOP $\wedge$ pipe2.op $\neq$ STORE $\wedge$ dest $^{\prime}=$ pipe2.dest $\rightarrow$ stall' $^{\prime}=$ true

[] default $\rightarrow$ stall' $:=$ false

Fig. 3. Pipeline stages 2 and 3, output, and stall 
We make use of this proposition as follows. First we decompose the specification $Q$ into its components $Q_{1}\|\cdots\| Q_{n}$. Then we find for each component $Q_{i}$ of the specification a suitable module $\Gamma_{i}$ (called an obligation module) and check that $\Gamma_{i} \preceq Q_{i}$. This is beneficial if the state space of $\Gamma_{i}$ is smaller than the state space of $P$. The module $\Gamma_{i}$ is the parallel composition of two kinds of modules-essential modules and constraining modules. The essential modules are chosen from the implementation $P$ so that every interface variable of $Q_{i}$ is an interface variable of some essential module. There may, however, be some external variables of $Q_{i}$ that are not observable for the essential modules. In this case, to ensure that $Q_{i}$ is refinable by $\Gamma_{i}$, we need to choose constraining modules from either from the implementation $P$ or from the specification $Q$ (other than $Q_{i}$ ). Once $Q_{i}$ is refinable by $\Gamma_{i}$, if the refinement check $\Gamma_{i} \preceq Q_{i}$ goes through, then we are done. Typically, however, the external variables of $\Gamma_{i}$ need to be constrained in order for the refinement check to go through. Until this is achieved, we must add further constraining modules to $\Gamma_{i}$.

It is preferable to choose constraining modules from the specification, which is less detailed than the implementation and therefore gives rise to smaller state spaces (in the undesirable limit, if we choose $\Gamma_{i}=P$, then the proof obligation $\Gamma_{i} \preceq Q_{i}$ involves the state space of $P$ and is no simpler than the original proof obligation $P \preceq Q$ ). Unfortunately, due to lack of detail, the specification often does not supply a suitable choice of constraining modules. According to the following simple property of the refinement relation, however, we can arbitrarily "enrich" the specification by composing it with new modules.

Proposition 4. [Abstraction modules] For all modules $P, Q$, and $A$, if $P \preceq Q \| A$ and $Q$ is refinable by $P$, then $P \preceq Q$.

So, before applying the assume-guarantee rule, we may add modules to the specification and prove $P \preceq Q\left\|A_{1}\right\| \cdots \| A_{k}$ instead of $P \preceq Q$. The new modules $A_{1}, \ldots, A_{k}$ are called $a b$ straction modules, as they usually give high-level descriptions for some implementation components, in order to provide a sufficient supply of constraining modules. In summary, the creativity required from the human verification expert is the construction of suilable abstraction modules, which on one hand, need to be as detailed as required to serve as constraining modules in assumeguarantee reasoning, and on the other hand, should be as abstract as possible to minimize their state spaces.

While witness modules are introduced "on the left" of a refinement relation, abstraction modules are introduced "on the right." So it may be necessary to iterate both processes, providing witnesses for abstractions, and abstractions for witnesses. An example of this will appear in the next section.

\section{Our Solution}

We prove that PIPELINE $\preceq I S A$ using Propositions $1,2,3$, and 4. We note that $I S A$ is refinable by PIPELINE, but not projection refinable. This is because isaRegFile in ISA is a private variable. We claim that the module ISARegFile is a witness module for isaRegFile. We then use Proposition 2 to reduce the proof obligation PIPELINE $\preceq I S A$ to ISARegFile\|PIPELINE $I S A^{u}$. This proof obligation can be expanded in terms of component modules to

ISARegFile $\|$ RegFile\|Opri\|Opr2\| Pipe1\|Pipe2\|PipeOut\|Stall $\preceq$ ISARegFile\|ISAOut\|ISAStall.

Let us start by identifying ISAOut with $Q_{1}$. We need to find an obligation module $\Gamma_{1}$, such that $\Gamma_{1} \preceq I S A$ Out. There is only one interface variable for ISAOut, namely out. The component of PIPELINE that generates out is PipeOut. Thus PipeOut is the only essential module for $\Gamma_{1}$. However, the proof obligation

$$
\Gamma_{1}=P_{i p e O u t} \preceq Q_{1}=I S A O u t
$$


fails trivially, because ISAOut is not refinable by PipeOut. The module ISAOut has an external variable isaRegFile that is not present in PipeOut. To achieve refinability, we add ISARegFile, the modulc controlling isaRegFile, to $\Gamma_{1}$ and try to prove

$$
\Gamma_{\mathrm{l}}=\text { ISARegFile\|PipeOut } \preceq Q_{1}=\text { ISAOut. }
$$

This fails because the input regFile to PipeOut is not constrained. We add RegFile to constrain regFile, but in vain, because the check

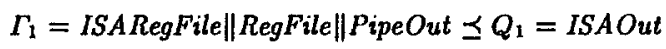

also fails. The reason now is that the inputs to RegFile are not constrained. We add Pipe2 for this purpose, and then Pipe1, Opr1, Opr2, and Stall to constrain the inputs to Pipe2. At last, we are able to prove the proof obligation

$$
\Gamma_{1}=\text { ISARegFile } \| \text { RegFile } \| \text { Pipe1 } \| \text { Pipe2 } \| \text { Opr1 } \| \text { Opr2\|Stall\|PipeOut } \preceq Q_{1}=\text { ISAOut. }
$$

Now, according to Proposition 3, the assume-guarantec proof looks as follows:

$$
\begin{aligned}
& \text { ISARegFile\|RegFile } \| \text { Pipe1\|Pipe2\| } \preceq \text { ISAOut } \\
& \text { Opr1\|Opr2\|Stall\|PipeOnt } \text { ISARegFile } \\
& \text { Stall ISARegFile } \preceq \text { ISAStall } \\
& \hline \text { ISARegFile\|RegFile\|Pipe1\|Pipe2\| } \\
& \text { Opr1\|Opr2\|Stall\|PipeOut } \preceq \text { ISAOut\|ISARegFile\|ISAStall }
\end{aligned}
$$

However, notice that the biggest module on the left side above the line is exactly the same as the module on the left side below the line. Hence, the compositional approach did not yield much advantage.

So let us return to the PIPELINE module with the intent of adding abstraction modules. We will add three abstraction modules-AbsOpr1, AbsOpr2, and AbsRegFile, corresponding to $O p r 1, O p r 2$, and RegFile. Notice that whenever the required operand specified by $s r c 1$ is currently being produced by $A L U$ or is in wbReg, module $O p r 1$ looks ahead and finds it. Otherwise, it gets the operand from the register file in PIPELINE. It is observed that the specification variable isaRegFile [src $1^{\prime}$ ] contains the same value that will be produced by the forwarding logic. This observation can be used to write the following abstraction module for Opr1.

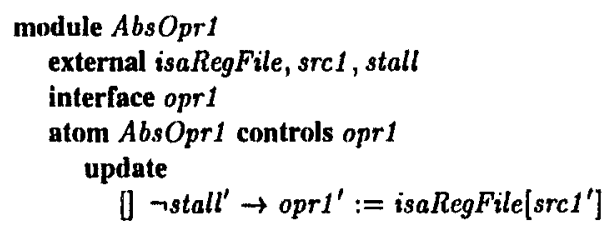

Note that the abstraction module lcaves the value of oprl unspecificd if stall is true. The implementation module $O p r 1$, on the other hand, specilics a value for opr 1 in every round. Such incomplete specification is an essential characteristic of abstraction modules. A similar abstraction module $A b s O p r 2$ can be written for Opr2.

To write an abstraction module for the implementation register file, regFile, observe that the vaiue of regFile in every round must be equal to the value of isaRegFile from two rounds earlier. Thus, we can write the abstraction module for RegFile as AbsRegFile\|ISARegFile ${ }_{d}$, where

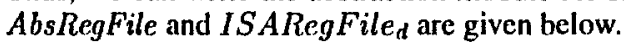

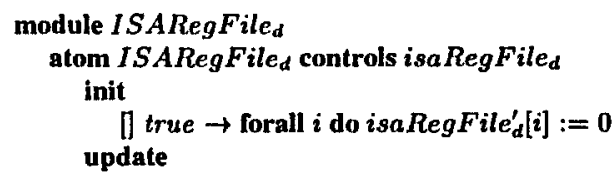




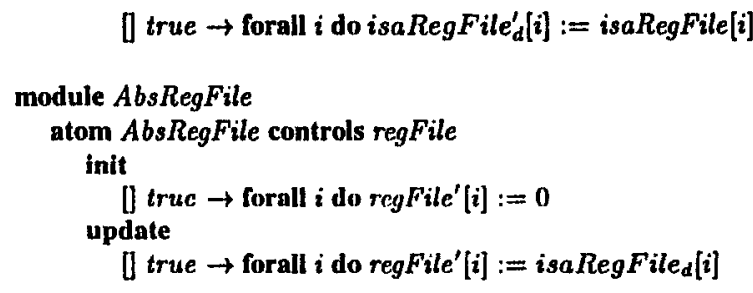

On composing AbsRegFile and ISARegFile ${ }_{d}$ with $I S A$, we find that the new specification is not projection refinable by ISARegFile $\mid P I P E L I N E$, because of the new specification variable

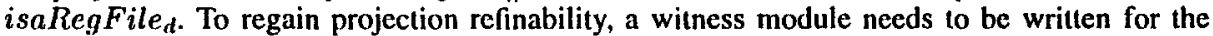
abstraction module isaRegFile $e_{d}$, and composed with PIPELINE. A suitable witness is simply the module ISARegFile. After adding the abstraction modules, aceording to Proposition 3 , we obtain the following assume-guarantee proof:

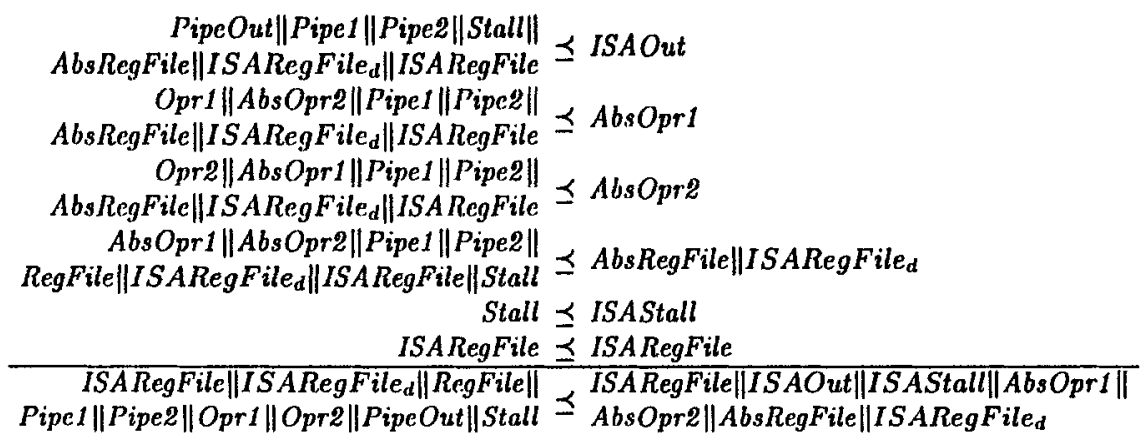

All proof obligations above the line satisfy projection refinability, and involve smaller state spaces than the conclusion of the proof. Following Proposition 1, they can be discharged by a transition-invariant check. Let us now focus on the modules below the line. Notice that the compositc module on the left side is PIPELINE \|ISARegFile \|ISARegFile , $_{\text {, }}$ and the composite modulc on the right side is $I S A^{u}\left\|I S A R e g F i l e_{\text {d }}\right\| A b s O_{p r 1\|A b s O p r 2\| A b s R e g F i l e . ~ B y}$ Proposition 4, we can remove ISARegFile $\left\|_{d} \mid A b s O p r 1\right\| A b s O p r 2 \| A b s R e g F i l e$ from the right side to obtain the refinement PIPELINE\|ISARegFile $\| I S A R e g F i l e_{d} \preceq I S A^{u}$. The module ISARegFile \|ISARegFiled is a witness for the refinement PIPELINE $I S A$. Hence, by Proposition 2, we conclude that PIPELINE $\preceq I S A$.

\section{Discussion}

In the previous section, we presented an assume-guarantee proof of the fact that PIPELINE refines $I S A$. In this section, we would like to touch upon some of the issues and finer points that came up while we were developing this methodology.

Projection refinability. Our definition of projection refinability is stronger than necessary. A variable is history-free if no atom uses the (latched) value of the variable from the previous round. Otherwise, the variable is said to be a latch variable. For module $Q$ to be projection refinable by module $P$, it is sufficient to require that every latch variable of $Q$ is obscrvable in both $P$ and $Q$.

Trivial witnesses. An atom is deterministic if two distinct guards of the initial command cannot be true in any given round, and the same is true for the update command. A module is deterministic if all its atoms are deterministic. If a private variable of the specification is controlled by a deterministic module, and all variables on which it depends are already present in the implementation, then the witness module for this variable can be easily constructed by copying the initial 
and update commands of the controlling module. This phenomenon can be noticed in the case study of Section 4, where we claimed ISARegFile as the witness for the variable isaRegFile. Notice also that this simplicity comes at a price. The module ISARegFile has latch variables, and so we have increased the number of state bits in the module over which we perform the transition-invariant check. Altcrnatively, a more complex witncss for isnRegFile, which does not have any latch variables, can be produced [HQR98].

Choice of constraining modules. $A n$ important problem one faces in a compositional proof is the choice of a mimimal set of constraining modules, preferably with small state spaces. Consider one proof obligation ("Iemma") $\Gamma_{i} \preceq Q_{i}$ in the compositional proof of $P \preceq Q$ using Proposition 3. Starting from the essential modules, our implementation chooses progressively larger obligation modules $\Gamma_{i}$ in two steps. First, sufficient constraining modules are added to make $Q_{i}$ refinable by $\Gamma_{i}$. Second, additional constraining modules are chosen according to a heuristics that looks at the data dependencies in the specification and implementation, until $\Gamma_{i}$ refines $Q_{i}$. The constraining modules are chosen preferably from the specification $Q$, rather than from the implementation $P$. Alternatively, the user can force specilic submodules of $P$ or $Q$ into $\Gamma_{\text {i }}$.

Fairness. Though not discussed here, our methodology also supports fairness conditions on the specification and implementation [HQR98].

\begin{tabular}{|l|c|}
\hline Refinement Check & Latches \\
\hline \hline Monolithic & $110^{*}$ \\
\hline msgP, indexP & 35 \\
msgBuffer & 39 \\
msgC, indexC & 59 \\
windowS & 75 \\
seqS & 35 \\
seqR & 59 \\
ackWait & 15 \\
seqX & 55 \\
msgX & 55 \\
ackX & 51 \\
busy & 75 \\
recvd & 56 \\
msgBufferR & 68 \\
\hline
\end{tabular}

\begin{tabular}{|l|c|}
\hline Refinement Check & Latches \\
\hline \hline Monolithic & $67^{*}$ \\
\hline Data Out & 12 \\
Bus valid bit & 0 \\
Bus value & 32 \\
Bus tag & 0 \\
Register[0] valid bit & 4 \\
Register[0] tag & 4 \\
Register[0] value & 20 \\
Reservation Station[0] valid bit & 4 \\
Reservation Station[0] aVal valid bit & 22 \\
Reservation Station[0] aVal tag & 10 \\
Reservation Station[0] aVal value & 35 \\
\hline
\end{tabular}

Table 1. Lemmas in the proof of sliding-window protocol (left) and Tomasulo's algorithm (right)

Other case studies. We used the methodology outlined in Section 3 to verify implementations of a sliding-window protocol and of Tomasulo's algorithm. Space does not permit us to describe these case studies in delail; a detailed description can be found in [HQR98]. The results of our experiments are summarized in Table 1. The table on the left gives the results for the slidingwindow protocol with window size 12. The table on the right gives the results for Tomasulo's algorithm with 4 registers and 4 reservation stations. The tables enumerate the lemmas that were proved to conclude that the implementation refines the specification. There is a lemma for cach component of the specification, and a lemma for each abstraction module that is composed with the specification. The second column gives the number of boolean latch variables that encode the state space of the corresponding obligation module. In all proofs, most obligation modules contained components from the specification or abstraction modules. These components are typically very abstract, with much nondeterminism and small state spaces. The row labeled "monolithic" refers to a noncompositional proof, where the transition-invariant check is performed on 
the full state space of the implementation. The superscript * indicates an unsuccessful verification attempt.

\section{Acknowledgments}

We thank Ken McMillan and Amir Pnueli for inspiring this work.

\section{References}

[AH96] R. Alur and T.A. Henzinger. Reactive modules. In Proceedings of the 11th Annual Symposium on Logic in Computer Science, pages 207-218. IEEE Computer Society Press, 1996.

$\left[\mathrm{AHM}^{+}\right.$98] R. Alur, T.A. Henzinger, F.Y.C. Mang, S. Qadeer, S.K. Rajamani, and S. Tasiran. MоснА : Modularity in model checking. In A. Hu and M. Vardi, editors, CAV 98: Computer Aided Verification, Lecture Notes in Computer Science. Springer-Verlag, 1998.

[AL91] M. Abadi and L. Lamport. The existence of refinement mappings. Theoretical Computer Science, 82(2):253-284, 1991.

[AL95] M. Abadi and L. Lamport. Conjoining specifications. ACM Transactions on Programming Languages and Systems, 17(3):507-534, 1995.

[BBLS92] S. Bensalem, A. Bouajjani, C. Loisenux, and J. Sifakis. Property-preserving simulations. In G. von Bochmann and D.K. Probst, cditors, CAV 92: Computer Aided Verification, Lecture Notes in Computer Science 663, pages 260-273. Springer-Verlag, 1992.

[CGL92] E.M. Clarke, O. Grumberg, and D.E. Long. Model checking and abstraction. In Proceedings of the 19th Anmual Symposium on Principles of Programming Languages, pages 343-354. ACM Press, 1992.

[CLM89] E.M. Clarke, D.E. Long, and K.L. McMillan. Compositional model checking. In Proceedings of the 4th Annual Symposium on Logic in Computer Science, pages 353-362. IEEE Computer Society Press, 1989.

[DP97] W. Damm and A. Pnueli. Verifying out-of-order executions. In Proceedings of the IFIP Working Conference on Correct Hardware Design and Verification Methods, CHARME, 1997.

[GL94] O. Grumberg and D.E. Long. Model checking and modular verification. ACM Transactions on Programming Languages and Syslems, 16(3):843-871, 1994.

[HQR98] T.A. Henzinger, S. Qadecr, and S.K. Rajamani. You assume, we guarantee: Mcthodology and case studies. Technical report, Electronics Research Lab, Univ. of California, Berkeley, CA 94720, 1998.

[Kur94] R.P. Kurshan. Computer-aided Verification of Coordinating Processes. Princeton University Press, 1994.

[Lam83] L. Lamport. Specifying concurrent program modules. ACM Transactions on Programming Languages and Systems, 5(2): 190-222, 1983.

[LT87] N.A. Lynch and M.R. Tuttle. Hicrarchical correctness proofs for distributed algorithms. In Procedings of the 6th Anmual Symposium on Principles of Distributed Computing, pages 137151. ACM Press, 1987.

[LV95] N.A. Lynch and F. Vaandrager. Forward and backward simulations, Part I: Untimed systems. Information and Computation, 121(2):214-233, 1995.

[McM97] K.L. McMillan. A compositional rule for hardware design refinement. In O. Gumberg, editor, CAV 97: Computer Aided Verification, Lecture Notes in Computer Science 1254, pages 24-35. Springer-Verlag, 1997.

[McM98] K.L. McMillan. Verification of an implementation of Tomasulo's algorithm by compositional model checking. In A. Hu and M. Vardi, editors, CAV 98: Computer Aided Verification, Lecture Notes in Computer Science. Springer-Verlag, 1998.

[Sta85] E. W. Stark. $\Lambda$ proof technique for rely/guarantec propertics. In Procedings of the Sth Conference on Foundations of Software Technology and Theoretical Computer Science, Lecture Notes in Computer Science 206, pages 369-391. Springer-Verlag, 1985.

['Tan92] Andrew S. Tanenbaum. Computer Networks. Prentice-Hall Inc., 1992. 